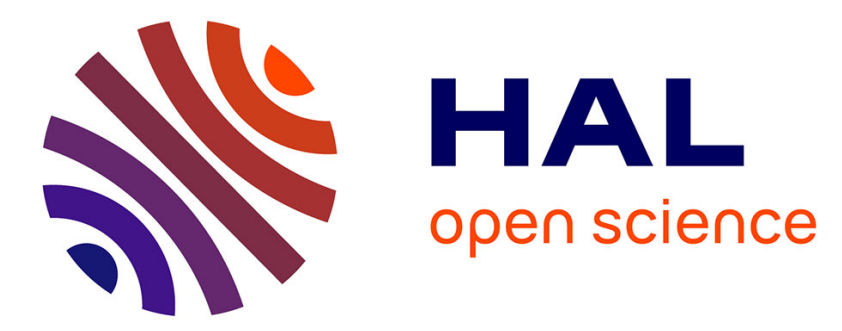

\title{
Estimation of state and unknown inputs of a non linear system represented by a multiple model
}

\author{
Abdelkader Akhenak, Mohammed Chadli, José Ragot, Didier Maquin
}

\section{To cite this version:}

Abdelkader Akhenak, Mohammed Chadli, José Ragot, Didier Maquin. Estimation of state and unknown inputs of a non linear system represented by a multiple model. 11th IFAC Symposium on Automation in Mining, Mineral and Metal processing, MMM 2004, Sep 2004, Nancy, France. pp.CDROM. hal-00151292

\section{HAL Id: hal-00151292 \\ https://hal.science/hal-00151292}

Submitted on 3 Jun 2007

HAL is a multi-disciplinary open access archive for the deposit and dissemination of scientific research documents, whether they are published or not. The documents may come from teaching and research institutions in France or abroad, or from public or private research centers.
L'archive ouverte pluridisciplinaire HAL, est destinée au dépôt et à la diffusion de documents scientifiques de niveau recherche, publiés ou non, émanant des établissements d'enseignement et de recherche français ou étrangers, des laboratoires publics ou privés. 


\title{
ESTIMATION OF STATE AND UNKNOWN \\ INPUTS OF A NONLINEAR SYSTEM REPRESENTED BY A MULTIPLE MODEL
}

\author{
A. Akhenak, M. Chadli, J. Ragot, D. Maquin \\ * Centre de Recherche en Automatique de Nancy, \\ CNRS UMR 7039. \\ Institut National Polytechnique de Lorraine. \\ 2, Avenue de la forêt de Haye. 54516 Vandoeuvre, France. \\ Email: aakhenak@ensem.inpl-nancy.fr
}

\begin{abstract}
In this paper, we consider a nonlinear system represented by a multiple model, where a part of its inputs is unknown. Our objective is to estimate the state variables and the unknown inputs of this system. For that, we propose the synthesis of a multiple observer based on the elimination of these unknown inputs. It is shown how to determine the gains of the local observers, these gains being solutions of a set of linear matrix inequalities (LMI). The model of an hydraulic system with three tanks is used to test the suggested procedure.
\end{abstract}

Keywords: Nonlinear system, unknown inputs, multiple model approach, multiple observer, asymptotic stability, linear matrix inequality.

\section{INTRODUCTION}

A physical process is often subjected to disturbances which have as origin the noises due to its environment, uncertainty of measurements, fault of sensors and/or actuators. These disturbances have harmful effects on the normal behavior of the process and their estimation can be used to conceive a control strategy able to minimize their effects. The disturbances are called unknown inputs when they affect the input of the process and their presence can make difficult the state estimation.

Several works were achieved concerning the estimation of the state and the output in the presence of unknown inputs. They can be gathered into two categories. The first one supposes an a priori knowledge of information on these nonmeasurable inputs; in particular, Johnson (Johnson, 1975) proposes a polynomial approach and Meditch (Meditch et al., 1971) suggests approximating the unknown inputs by the response of a known dynamic system. The second category proceeds either by estimation of the unknown inputs, or by their complete elimination from the equations of the system.

Among the techniques that do not require the elimination of the unknown inputs, that of Wang (Wang et al., 1975) proposes an observer able to entirely reconstruct the state of a linear system in the presence of unknown inputs; Kobayashi (Koba et al., 1982) and Lyubchik (Lyubchik et al., 1993) use a method of model inversion to estimate the state.

Among the techniques which allow the elimination of the unknown inputs, that of Kudva (Kudva et al., 1980) establishes, in the case of linear systems, the existence conditions of the system observer with unknown inputs while being based on the technique of generalized inverse of matrix. Guan carried out the elimination of the unknown in- 
puts of the state equations of a continuous linear system (Guan et al., 1991). Many of other alternatives exist, but most of them were developed principally for linear systems.

However, the real physical systems are often nonlinear. As it is delicate to synthesize an observer for a nonlinear system, we preferred to represent these systems with a multiple model. The idea of the multiple model approach is to apprehend the total behavior of a system by a set of local models (linear or affine), each local model characterizing the behavior of the system in a particular zone of operation. The local models are then aggregated by means of an interpolation mechanism.

The motivation of this approach rises owing to the fact that it is often difficult to design a model which takes into account all the complexity of the studied system. In 1985, Takagi and Sugeno (Takagi et al., 1985), presented their fuzzy model of a system described by a set of rules " if premise then consequence", such as the consequence of a rule is an affine local model; the global model is obtained by the sum of the local models weighted by activation functions associated to each of them.

For state estimation, the suggested technique consists in associating to each local model a local observer. The global observer (multiple observer) is the sum of the local observers weighted by their activation functions, which are the same than those associated with the local models (Patton et al., 1998). Our contribution lies in the design of this global observer by eliminating the unknown inputs from the system. The stabilization of the multiple observer is performed by the search of suitable Lyapunov matrices and the improvement of the performances of the multiple observer by pole assignment is formulated in a LMI context.

\section{ESTIMATION OF STATE AND UNKNOWN INPUT OF A MULTIPLE MODEL}

This section clarifies the construction of the observer. This last has an analytical form resulting from the aggregation of local observers and this form is particularly suitable for stability and convergence study of the estimation error. The numerical aspects concerning the determination of the gains of the observers will be also treated. A state observer can reconstruct the state of a dynamic system and has important applications such as the realization of the command by feedback, the monitoring system, and the diagnosis of faults. The Luenberger observer and it's variants are well known in the literature of control. The Luenberger observer works well when the dynamics of the system is known. The state reconstruction of a uncertain system or subjected to the influence of the unknown inputs is a classic problem of the automatic. However, the Luenberger observer is not always adapted for the fault detection, because the state estimation error given by this observer for a system with unknown inputs does not converge necessarily towards zero.

To improve these results within the framework of linear systems, we can use observers of singular systems (Lewis, 1986) or observers with unknown inputs (?). In the linear system framework, observers can be designed for singular systems, unknown input systems, delay systems and also uncertain system with time-delay perturbations (Fan et al., 2002). In the case of nonlinear system with unknown inputs represented by multiple model we have already proposed a technique for the state estimation of this multiple model by using a multiple observer with sliding mode (Akh et al., 2003). Within the framework of the hybrid systems one can cite (Alessandri et al., 2001) and (Balucchi et al., 2001).

In this work, we consider the estimation of the state vector and the unknown inputs of a nonlinear system represented by a multiple model and subject to the influence of unknown inputs, by using a multiple observer. This multiple observer is based on local Luenberger observers including a sliding term to compensate the effect of the unknown inputs.

We also present a criterion of stability of this multiple observer according to Lyapunov's approach (Chadli et al., 2003) and the LMI approach (Boyd et al., 1994). Let us consider a nonlinear system represented by the following multiple model (with $M$ local models) subject to unknown inputs:

$$
\begin{aligned}
& \left\{\begin{array}{l}
\dot{x}=\sum_{i=1}^{M} \mu_{i}(\xi)\left(A_{i} x+B_{i} u+R_{i} \bar{u}+D_{i}\right) \\
y=C x
\end{array}\right. \\
& \text { such that: }\left\{\begin{array}{l}
\sum_{i=1}^{M} \mu_{i}(\xi)=1 \\
0 \leq \mu_{i}(\xi) \leq 1 \quad \forall i=\{1, \ldots, M\}
\end{array}\right.
\end{aligned}
$$

where $x(t) \in \Re^{n}$ is the state vector, $u(t) \in \Re^{m}$ is the input vector, $\bar{u}(t) \in \Re^{q}, q<n$, the vector of unknown inputs and $y(t) \in \Re^{q}$ the vector of measurable output. For the $i^{\text {th }}$ local model $A_{i} \in \Re^{n \times n}$ is the state matrix, $B_{i} \in$ $\Re^{n \times m}$ is the matrix of input, $R_{i} \in \Re^{n \times q}$ is the matrix of influence of the unknown inputs and $D_{i} \in \Re^{n \times 1}$ is a matrix depending on the operating point. Finally, $C \in \Re^{p \times n}$ is the matrix of output. $\xi$ represents the vector of decision depending on the input and/or the measurable state variables. The obtention of the multiple model is out of purpose in this presentation but for the interested reader, let us state that one can either uses techniques of parametric estimation 
(Gasso et al., 2001) or techniques of linearization (Johanson et al., 2000).

The problem considered here is those of the reconstruction of the state variable by using only the information contained in the known inputs and the measurable outputs.

\subsection{General structure of the multiple observer}

The proposed observer for the multiple model (1) is a linear combination of local observers, each of them having the structure proposed by Walcott and Zak. In this part, we consider that the inputs $\bar{u}(t)$ are bounded, such as $\|\bar{u}(t)\| \leq \rho$, where $\rho$ is scalar and $\|\cdot\|$ represents the Euclidean norm. It is also assumed that there exists matrices $G_{i} \in \Re^{n \times p}$, such that $A_{0 i}=A_{i}-G_{i} C$ have stable eigenvalues and that there exists Lyapunov pairs $\left(P, Q_{i}\right)$ of matrices and matrices $F_{i} \in \Re^{m \times p}$ respecting the following structural constraints:

$$
\left\{\begin{aligned}
A_{0 i}^{T} P+P A_{0 i} & =-Q \\
C^{T} F_{i}^{T} & =P R_{i}, \quad \forall i \in 1, \ldots, M
\end{aligned}\right.
$$

The proposed observer has the form:

$$
\left\{\begin{array}{l}
\dot{\hat{x}}=\sum_{i=1}^{M} \mu_{i}(\xi)\left(A_{i} \hat{x}+B_{i} u+G_{i} e+R_{i} \nu_{i}\right) \\
y=C \hat{x}
\end{array}\right.
$$

where $e(t)$ represent the state estimation error, such as:

$$
e(t)=x(t)-\hat{x}(t)
$$

The matrices $G_{i}$ and the control variables $\nu_{i}$, with $\nu_{i}(t) \in \Re^{q}$ must be determined in order to guarantee the asymptotic convergence of $\hat{x}(t)$ towards $x(t)$. The terms $\nu_{i}(t)$ compensate errors due to the unknown inputs. The set of residuals is defined as follows:

$$
r=y-\hat{y}=C(x-\hat{x})=C e
$$

The dynamic of state estimation error is given as follows:

$$
\dot{e}=\sum_{i=1}^{M} \mu_{i}(\xi)\left(\left(A_{i}-G_{i} C\right) e+R_{i} \bar{u}-R_{i} \nu_{i}\right)
$$

Theorem 1: The state estimation error between the multiple model (1) and the robust state multiple observer (3) converges towards zero, if $\nu_{i}(t)$ are given by the following equation:

$$
\left\{\begin{array}{l}
\text { if } r(t) \neq 0, \text { then } \nu_{i}(t)=\rho \frac{F_{i} r(t)}{\left\|F_{i} r(t)\right\|} \\
\text { if } r(t)=0, \text { then } \nu_{i}(t)=0
\end{array}\right.
$$

and if there exists a symmetric definite positive matrix $P$ which satisfies the following inequalities:

$$
\begin{gathered}
\left(A_{i}-G_{i} C\right)^{T} P+P\left(A_{i}-G_{i} C\right)<0, \\
i=\{1, \ldots, M\} .
\end{gathered}
$$

Proof: In order to show the asymptotic convergence of this multiple observer, let us consider the following Lyapunov function:

$$
V(e(t))=e^{T}(t) P e(t)
$$

Its derivative in respect to time, evaluated along the trajectory of the system by using equations (5) and (6), may be expressed as:

$$
\begin{aligned}
\dot{V}=\sum_{i=1}^{M} \mu_{i}(\xi) & \left(e^{T}\left(\bar{A}_{i}^{T} P+P \bar{A}_{i}\right) e\right. \\
& \left.+2 e^{T} P R_{i} \bar{u}-2 e^{T} P R_{i} \nu_{i}\right)
\end{aligned}
$$

where $\bar{A}_{i}=A_{i}-G_{i} C$.

Using the second part of constraint (2), the derivative of the Lyapunov function becomes:

$$
\begin{aligned}
\dot{V}=\sum_{i=1}^{M} \mu_{i}(\xi) & \left(e^{T}\left(\bar{A}_{i}^{T} P+P \bar{A}_{i}\right) e\right. \\
& \left.+2 e^{T} C^{T} F_{i}^{T} \bar{u}-2 e^{T} C^{T} F_{i}^{T} \nu_{i}\right) \\
=\sum_{i=1}^{M} \mu_{i}(\xi) & \left(e^{T}\left(\bar{A}_{i}^{T} P+P \bar{A}_{i}\right) e\right. \\
& \left.+2 r^{T} F_{i}^{T} \bar{u}-2 r^{T} F_{i}^{T} \nu_{i}\right) \\
\leq \sum_{i=1}^{M} \mu_{i}(\xi) & \left(e^{T}\left(\bar{A}_{i}^{T} P+P \bar{A}_{i}\right) e\right. \\
& \left.+2 \rho\left\|F_{i} r\right\|-2 r^{T} F_{i}^{T} \nu_{i}\right)
\end{aligned}
$$

Using the relation (7), the derivative of the Lyapunov function becomes as follows:

$$
\begin{aligned}
& \dot{V} \leq \sum_{i=1}^{M} \mu_{i}(\xi)\left(e^{T}\left(\bar{A}_{i}^{T} P+P \bar{A}_{i}\right) e+\right. \\
& \left.2 \rho\left\|F_{i} r\right\|-2 \rho r^{T} F_{i}^{T} \frac{F_{i} r}{\left\|F_{i} r\right\|}\right) \\
& \dot{V} \leq \sum_{i=1}^{M} \mu_{i}(\xi)\left(e^{T}\left(\bar{A}_{i}^{T} P+P \bar{A}_{i}\right) e\right)
\end{aligned}
$$

Then, the state estimation error of the robust multiple observer (3) converges towards zero if the relation (8) holds.

\subsection{Unknown input estimation}

Several works were realized for the unknown input estimation within the framework of linear dynamic systems (Edwards et al., 2000). The original method developed in this paper, is based on the knowledge of matrices $A_{i}, B_{i}, D_{i}, R_{i}$ and the output matrix $C$ of the multiple model (1). By knowing these matrices, one can suppose the existence of a fictitious multiple model of the following form:

$$
\left\{\begin{array}{l}
\dot{\bar{x}}=\sum_{i=1}^{M} \mu_{i}(\xi)\left(A_{i} \bar{x}+B_{i} u+D_{i}\right) \\
\bar{y}=C \bar{x}
\end{array}\right.
$$

We have previously shown that the convergence of the multiple observer is guaranteed if the condition (8) and the constraints (2) are verified. In 
steady state regime, the state estimation error towards zero, then $x(t)=\hat{x}(t)$; by replacing $\hat{x}(t)$ in the equation (1) we obtain:

$$
\left\{\begin{array}{l}
\dot{\hat{x}}=\sum_{i=1}^{M} \mu_{i}(\xi)\left(A_{i} \hat{x}+B_{i} u+R_{i} \hat{\bar{u}}+D_{i}\right) \\
y=C \hat{x}
\end{array}\right.
$$

where $\hat{\bar{u}}(t)$ represent the unknown inputs that are to be estimated. We consider $\varepsilon(t)$ the fictitious state estimation error between the system (12) and fictitious system (13), given as follows:

$$
\varepsilon=\hat{x}-\bar{x}
$$

The dynamic of this fictitious state estimation error is given by the following equation:

$$
\dot{\varepsilon}=\dot{\hat{x}}-\dot{\bar{x}}=\sum_{i=1}^{M} \mu_{i}(\xi)\left(A_{i} \varepsilon+R_{i} \hat{\bar{u}}\right)
$$

So, assuring the existence the generalized inverse of the transmission matrix of the unknown inputs $\hat{\bar{u}}(t)$ allows to give estimation:

$$
\hat{\bar{u}}=\left(\sum_{i=1}^{M} \mu_{i}(\xi) R_{i}\right)^{-}\left(\dot{\varepsilon}-\sum_{i=1}^{M} \mu_{i}(\xi) A_{i} \varepsilon\right)
$$

\subsection{Design of a multiple observer with relaxed constraints}

To establish the existence conditions of a multiple observer for the multiple model subjected to the influence of the unknown inputs, another method may be proposed. The multiple observer has now the following form:

$$
\left\{\begin{array}{l}
\dot{\hat{x}})=\sum_{i=1}^{M} \mu_{i}(\xi)\left(A_{i} \hat{x}+B_{i} u+G_{i} e+\nu_{i}\right) \\
\hat{y}=C \hat{x}
\end{array}\right.
$$

It is necessary to note that the terms $\nu_{i}(t)$ compensate the errors due to the unknown inputs. In order to determine the matrices $G_{i}$ and the terms $\nu_{i}(t) \in \Re^{p}$ which guarantee the asymptotic convergence of $\hat{x}(t)$ towards $x(t)$, let us define the state and the output estimation error:

$$
\begin{gathered}
e=x-\hat{x} \\
r=y-\hat{y}=C(x-\hat{x})=C e
\end{gathered}
$$

The dynamic of state estimation error is given by the following equation:

$$
\dot{e}=\sum_{i=1}^{M} \mu_{i}(\xi)\left(\left(A_{i}-G_{i} C\right) e+R_{i} \bar{u}-\nu_{i}\right)
$$

Lemma 1: For any matrices $X$ and $Y$ with appropriate dimensions, the following property holds:

$$
X^{T} Y+Y^{T} X \leq \beta X^{T} X+\beta^{-1} Y^{T} Y \text {, with } \beta>0
$$

Theorem 2: The state estimation error between the multiple model (1) and the robust multiple observer (17) converges towards zero, if $\nu_{i}(t)$ are given as follows:

$$
\left\{\begin{array}{l}
\text { if } r \neq 0, \text { then } \nu_{i}=\frac{\rho^{2} \beta^{-1}\left\|P R_{i}\right\|^{2}}{2 r^{T} r} P^{-1} C^{T} r \\
\text { if } r=0, \text { then } \nu_{i}=0
\end{array}\right.
$$

with $\beta$ a positive scalar. $P$ is a symmetric and positive definite matrix which satisfies the following inequalities:

$$
\begin{gathered}
\left(A_{i}-G_{i} C\right)^{T} P+P\left(A_{i}-G_{i} C\right)+\beta I<0 \\
i=\{1, \ldots, M\}
\end{gathered}
$$

Proof: In order to show the asymptotic convergence of this multiple observer, let us consider the following Lyapunov function:

$$
V(e(t))=e^{T}(t) P e(t)
$$

Its derivative in respect to time, evaluated along the trajectory of the system by using equations (19) and (20), may be expressed as:

$$
\begin{array}{r}
\dot{V}=\sum_{i=1}^{M} \mu_{i}(\xi)\left(e^{T}\left(\bar{A}_{i}^{T} P+P \bar{A}_{i}\right) e+\right. \\
\left.2 e^{T} P R_{i} \bar{u}-2 e^{T} P \nu_{i}\right)
\end{array}
$$

It is easy to verify that: $2 e^{T} P R_{i} \bar{u}=e^{T} P R_{i} \bar{u}+$ $\bar{u}^{T} R_{i}^{T} P e$ and by using the lemma (1), we obtain the following inequalities:

$$
\begin{aligned}
e^{T} P R_{i} \bar{u}+\bar{u}^{T} R_{i}^{T} P e & \leq \beta e^{T} e+\beta^{-1} \bar{u}^{T} R_{i}^{T} P P R_{i} \bar{u} \\
& \leq \beta e^{T} e+\beta^{-1}\left\|P R_{i} \bar{u}\right\|^{2} \\
& \leq \beta e^{T} e+\beta^{-1} \rho^{2}\left\|P R_{i}\right\|^{2}
\end{aligned}
$$

By using the relation (22), the fact that the unknown inputs are bounded and the properties of the lemma (1), the derivative of the Lyapunov function may be bounded as follows:

$$
\begin{gathered}
\dot{V} \leq \sum_{i=1}^{M} \mu_{i}(\xi)\left(e^{T}\left(\bar{A}_{i}^{T} P+P \bar{A}_{i}+\beta I\right) e+\right. \\
\left.\beta^{-1} \rho^{2}\left\|P R_{i}\right\|^{2}-\rho^{2} \beta^{-1}\left\|P R_{i}\right\|^{2} e^{T} \frac{C^{T} r}{r^{T} r}\right) \\
\leq \sum_{i=1}^{M} \mu_{i}(\xi)\left(e^{T}\left(\bar{A}_{i}^{T} P+P \bar{A}_{i}+\beta I_{n \times n}\right) e\right)
\end{gathered}
$$

Thus, the state estimation error converges asymptotically to zero, if the conditions (22) and the inequalities (23) are verified. Note that inequalities (23) depend on the two unknown matrices $P$ and $G_{i}$ which can be obtained by using LMI techniques.

\section{SIMULATION EXAMPLE}

The chosen nonlinear system is represented on figure 1. It results from a traditional benchmark (Zolghadri et al., 1996) and schematizes a hydraulic process made up of three tanks. These three tanks $T_{1}, T_{2}$ and $T_{3}$, with identical sections 
$A$, are connected one to each other by cylindrical pipes with identical sections $S_{n}$. The output valve is located at the output of tank $T_{2}\left(T_{2}\right.$ ensures to empty the tank filled by the pump flows 1 and 2 with respective rates $Q_{1}(t)$ and $\left.Q_{2}(t)\right)$. Two combinations of the three water levels are measured. The communication pipes between the tanks are equipped with manually adjustable ball valves, which allow the corresponding pump to be closed or open. The three levels are governed by the constraint $x_{1}>x_{3}>x_{2}$; the process model is given by equations (26). Indeed, taking into account the fundamental laws of conservation of the fluid, one can describe the operating mode of each tank; one then obtains a nonlinear model expressed by the following state equations:

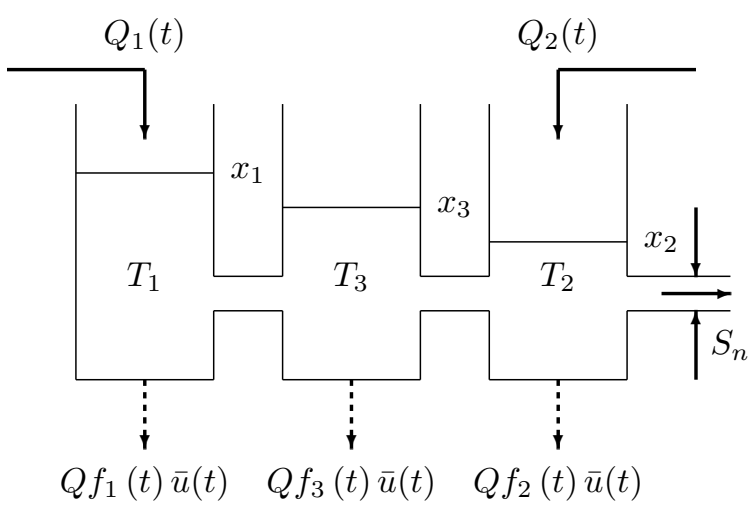

Figure 1. Three tank system

$$
\left\{\begin{array}{c}
A \frac{d x_{1}}{d t}=Q_{1}-\alpha_{1} S_{n} \sqrt{2 g\left(x_{1}-x_{3}\right)}+Q f_{1} \bar{u} \\
A \frac{d x_{2}}{d t}=Q_{2}+\alpha_{3} S_{n} \sqrt{2 g\left(x_{3}-x_{2}\right)}- \\
\alpha_{2} S_{n} \sqrt{2 g x_{2}}+Q f_{2} \bar{u} \\
A \frac{d x_{3}}{d t}=\alpha_{1} S_{n} \sqrt{2 g\left(x_{1}-x_{3}\right)}- \\
\alpha_{3} S_{n} \sqrt{2 g\left(x_{3}-x_{2}\right)}+Q f_{3} \bar{u}
\end{array}\right.
$$

where $\alpha_{1}, \alpha_{2}$ and $\alpha_{3}$ are constants. $Q f_{i}(t) \bar{u}(t), i \in$ $\{1, \ldots, 3\}$ denotes additional mass flows caused by leaks. The vector $\bar{u}(t)$ constitutes the unknown input. $g$ is the gravity constant and the numerical application are performed with:

$$
\left\{\begin{array}{l}
g=980 \mathrm{~cm} / \mathrm{sec}^{2}, A=154 \mathrm{~cm}^{2}, S_{n}=0.5 \mathrm{~cm}^{2} . \\
\alpha_{1}=0.9, \alpha_{2}=0.6, \alpha_{3}=0.8
\end{array}\right.
$$

The multiple model proposed (1), with $\xi(t)=$ $u(t)$, which approximates the nonlinear system $(26)$, is described by:

$$
\left\{\begin{array}{l}
\dot{x}=\sum_{i=1}^{2} \mu_{i}(\xi)\left(A_{i} x+B_{i} u+R_{i} \bar{u}+D_{i}\right) \\
y=C x
\end{array}\right.
$$

The matrices $A_{i}, B_{i}, R_{i}, C$ et $D_{i}$ are calculated by linearizing the initial system (26) around different points chosen in the operating range of the system. Two local models have been selected in an heuristic way. That number guarantees a good approximation of the state of the real system by the multiple model. The numerical values of all these matrices are:

$$
\begin{gathered}
A_{1}=10^{-3}\left[\begin{array}{ccc}
-18.5 & 0 & 18.5 \\
0 & -20.9 & 15.0 \\
18.5 & 15.0 & -33.5
\end{array}\right] \\
A_{2}=10^{-3}\left[\begin{array}{ccc}
-22.1 & 0 & 22.1 \\
0 & -23.3 & 17.6 \\
22.1 & 17.6 & -39.5
\end{array}\right] \\
R_{1}=10^{-3}\left[\begin{array}{l}
-0.57 \\
-0.46 \\
-0.52
\end{array}\right], R_{2}=10^{-3}\left[\begin{array}{l}
-0.57 \\
-0.50 \\
-0.54
\end{array}\right] \\
B_{1}=B_{2}=\frac{1}{154}\left[\begin{array}{lll}
1 & 0 & 0 \\
0 & 1 & 0
\end{array}\right]^{T} \\
D_{1}=\left[\begin{array}{c}
-0.225 \\
-0.089 \\
0.005
\end{array}\right], D_{2}=\left[\begin{array}{c}
-0.182 \\
-0.141 \\
0.003
\end{array}\right]
\end{gathered}
$$

The Lyapunov matrix $P$ and the two matrices $F_{1}$ and $F_{2}$ have been obtained by solving (8) and the second part of contraints (2):

$$
\begin{aligned}
& P=10^{2}\left[\begin{array}{ccc}
3.32 & -0.04 & 3.14 \\
-0.04 & 6.41 & -0.16 \\
3.14 & -0.16 & 3.45
\end{array}\right] \\
& F_{1}=[-0.29-0.07] \quad F_{2}=[-0.31-0.05]
\end{aligned}
$$

The simulation results are on the following figures:

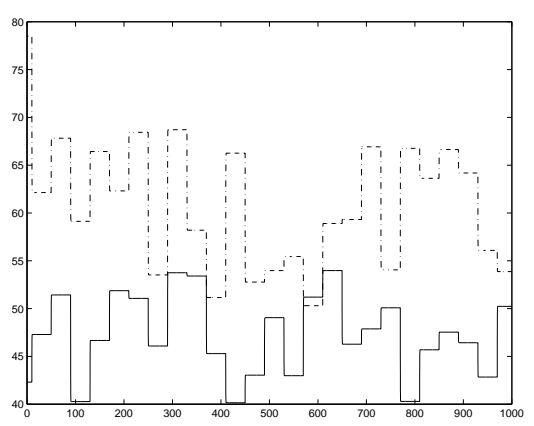

Figure 2. The inputs $\left(Q_{2}(t)-.-\right.$, and $\left.Q_{1}(t),-\right)$

\section{CONCLUSION}

From a multiple model representation, we show how to design a multiple observer using the principle of the interpolation of local observers; moreover, we have considered the case where certain inputs of the system were unknown. The calculation of the gains of the multiple observer is then returned to a calculation of gains of the local observers. The stability of the global observer requires however the consideration of coupling constraints between these local observers; 


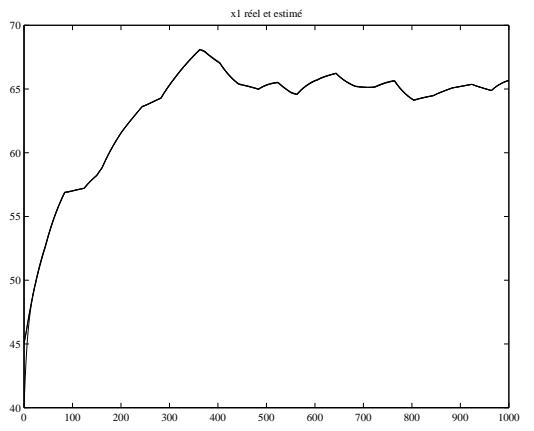

Figure 3. State $x_{2}(t)$ (true value, value given by the multiple model, value given by the multiple observer)

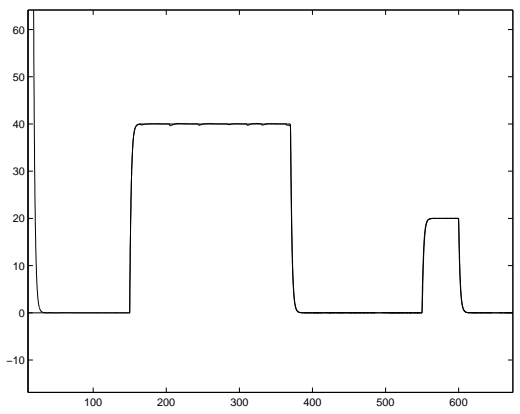

Figure 4. Unknown input and its estimate

these contraints lead to the resolution of a LMI problem under structural constraints. Assuming the existence of suited matrices, we showed that the reconstruction of the state vector and the unknown inputs of the multiple model is possible. The results of simulation show that the estimation of state and unknown inputs is very satisfactory.

\section{REFERENCES}

Boyd S., El Ghaoui L., Feron E. and Balakrishnan V. (1994). Linear Matrix Inequalities in System and Control Theory. Philadelphia: SIAM.

Lewis F. L. (1986). A survey of linear singular systems. Circuits systems signal process. Vol. 5 . n. 1. pp. 3-36.

Akhenak A. Chadli M. Maquin D. and Ragot J. (2003). Sliding mode multiple observer for fault detection and isolation. In proc. Of the 42th IEEE CDC. Haway.

Fan K. K. and Hsieh J. G. (2002). LMI Approach to design of robust state observer for uncertain systems with time-delay perturbation. IEEE ICIT'02. Vol. 42. pp. 1111-1115. Bongkok.

Zolghadri A. Henry D. Monsion M. (1996). Design of nonlinear observers for fault diagnosis: a case study. Control Engineering Practice. Vol. 4. n. 11. pp. 1535-1544.

Chadli M. Maquin D. Ragot J. (2003). On stability analysis of a class of multiple model. In proc. Of the 42th IEEE CDC. Haway.
Meditch J. S. and Hostetter G. H. (1971). Observers for systems with unkonwn and inaccessible inputs. Int. J. Control. Vol. 19. . pp. 637640.

Kobayashi N. and Nakamizo T. (1982). An observer design for linear systems with unknown inputs. Int. J. Control. Vol. 35. pp. 605-619.

Johnson C. D. (1975). Observers for linear systems sith unknown and inaccessible inputs. Int. J. Control. Vol. 21. pp. 825-831.

Kudva P. Viswanadham N. and Ramakrishna A. (1980). Observers for linear systems with unknown inputs. On Automatic Control. Vol. 25. pp. 113-115.

Lyubchik L. M. and Kostenko Y. T. (1993). he output control of multivariable systems with unmeasurable arbitrary disturbances - The inverse model approach. ECC'93. pp. 1160-1165. Netherlands.

Guan Y. and Saif M. (1991). A novel approach to the design of unknown input observers. IEEE trans on Automatic Control. Vol. 36. n. 5. pp. 632-635.

Johansen T. A. Shorten R. and Murray-Smith R. (2000). On the interpretation and identification dynamic Takagi-Sugeno fuzzy models. IEEE Trans on Fuzzy Systems. Vol. 8. n. 3. pp. 297-313.

Gasso K. Mourot G. and Ragot J. (2001). Structure identification in multiple model representation: elimination and merging of local models. IEEE Conference on Decision and Control. USA.

Patton R. J. and Chen J. and Lopez - Toribio C. J. (1998). Fuzzy observer for nonlinear dynamic systems. IEEE Conference on Decision and Control. Tampa Florida.

Johansen T. A. Shorten R. and Murray-Smith R. (1985). Fuzzy identification of systems and its application to moddeling and control. IEEE trans on Systems Man and Cybernetics-part C. Vol. 15. n. 1. pp. 116-132.

Wang S. H. Davison E. J. and Dorato P. (1975). Observing the states of systems with unmeasurable disturbances. IEEE Trans on Automatic Control. Vol. 20. pp. 716-717.

Edwards C. and Spurgeon S. K. (2000).Sliding mode observers for fault detection and isolation. Automatica. Vol. 36. n. 4. pp. 541-553.

A. Alessandri and P. Coletta (2001). Design of Luenberger observers for a class of hybrid linear systems. in Hybrid Systems: Computation and Control, Lecture Notes in Computer Science, Springer, pp. 7-18.

A. Balucchi, L. Benvenuti, M. D. Di Benedetto, and A. Sangiovanni-Vincentelli (2002). Design observers for hybrid systems. in Hybrid Systems: Computation and Control, Lecture Notes in Computer Science, Springer, pp. 76-89. 